\author{
Laurent Kodjikian \\ Jean-Daniel Grange \\ Stéphano Baldo \\ Stéphanie Baillif \\ Justus G. Garweg \\ Michel Rivoire
}

\section{Prognostic factors of liver metastases from uveal melanoma}

Received: 8 December 2004

Revised: 24 March 2005

Accepted: 29 March 2005

Published online: 13 May 2005

(C) Springer-Verlag 2005

A summary of this work was presented at the 27th congress of the "Societa Italiana di Chirurgia Oncologica" (May 29-31, 2003) and published as an abstract

This work was presented at the 24th

Meeting of the Club Jules Gonin,

September 2004, Athens (Greece)

Laurent Kodjikian had full access to all the data and takes responsibility for the integrity of the data in the study and the accuracy of the data analysis

L. Kodjikian $(\bowtie) \cdot$ J.-D. Grange ·

S. Baillif

Department of Ophthalmology,

Croix-Rousse Hospital,

103, Grande rue de la Croix-Rousse, Lyon, 69004, France

e-mail: kodjikian.laurent@wanadoo.fr

Tel.: +33-472-071718

Fax: +33-472-071708

L. Kodjikian · J. G. Garweg

Department of Ophthalmology,

Inselspital, Bern University,

Bern, Switzerland

S. Baldo · M. Rivoire

Department of Surgery,

Centre Léon Bérard,

Lyon, France
Abstract Objectives: This study was designed to assess survival and identify prognostic factors for liver metastases diagnosed by systematic screening in uveal melanoma patients. Methods: Among 602 consecutive patients treated over 10 years for uveal melanoma and followed by systematic semi-annual hepatic screening (abdominal ultrasonography), 63 (10.5\%) developed liver metastases; these patients form the basis of this study. Factors including patient demographics, characteristics of the uveal tumor, metastasis-free interval, severity of liver metastatic involvement, and treatments of metastases were studied retrospectively regarding their prognostic value, using univariate (Kaplan-Meier method) and multivariate (Cox model) analyses. Results: Thirtyfive patients $(55.6 \%$ of the metastatic population) received systemic chemotherapy or best supportive care only; 14 patients $(22.2 \%$ of the metastatic population) diagnosed with diffuse liver involvement had cytoreductive surgery and intra-arterial chemotherapy; 14 (22.2\% of the metastatic population) had complete surgical removal of liver metastases followed by postoperative intra-arterial chemotherapy. No significant surgical complications were experienced. The median overall survival after diagnosis of liver metastases was 15 months. It reached 25 months for selected patients with complete resection $(P=0.0002)$. In this cohort of 63 patients, ten or fewer preoperatively diagnosed metastases and primary uveal melanoma not involving the ciliary body were independently associated with better prognosis. Conclusions: This study suggests that selected patients with screened liver metastases from uveal melanoma may benefit from aggressive treatment, including surgery. The two independent favorable prognostic factors are fewer than ten metastases at screening and the absence of ciliary body involvement.

Keywords Uveal melanoma - Liver metastasis - Surgical treatment . Prognostic factors

\section{Introduction}

Uveal melanoma is the most common primary intraocular malignancy in adults. It arises from uveal melanocytes residing in the uveal stroma, and accounts for $70 \%$ of all primary ocular cancers [29]. The incidence of uveal melanoma in the French population has been estimated to be 0.73 per 100,000 , its overall prevalence being 7.4 per $100,000[30,34]$. Metastases from uveal melanoma occur in a significant number of treated patients $(6.5-35 \%)[7,8$, 
21]. For most patients, the liver is the primary and only site involved at the time of detection of systemic metastases [18]. Prognosis is reported to be very poor, depending above all on liver involvement, since no systemic treatment has been proven to be effective $[2,5,6,11]$. Only a few prognostic factors for survival have been identified. Age, short time interval between diagnosis of primary uveal melanoma and metastasis, and importance of liver metastatic burden have shown a negative impact on survival, whereas patients diagnosed at regular follow-up survived significantly longer $[3,5,6,11,13,23,25]$. It is well established nowadays that screening examination is advocated for all patients affected by uveal melanoma $[5,6]$. Therefore, criteria regarding the severity of liver metastatic involvement [poor performance status, elevated percentage of hepatic replacement, large metastasis diameter, and abnormalities in liver function tests (LFTs)] no longer seem to be applicable. Moreover, most published series have been biased due to patient selection criteria.

In the present study, we retrospectively reviewed the files of a complete series of consecutive patients treated at a single institution for uveal melanoma, in order to identify prognostic factors and to evaluate survival in patients with liver metastases detected by systematic screening. We included both previously studied factors and new ones, especially uveal tumor characteristics.

\section{Patients and methods}

\section{Patients}

Between June 1, 1983 and December 31, 1996, 602 consecutive patients suffering from primary uveal melanoma were registered in the Department of Ophthalmology at Croix-Rousse Hospital, Claude Bernard University, in Lyon, France. All patients were followed at regular 6-month intervals with clinical examination and ocular and abdominal ultrasonography. The compliance with the follow-up was high, with an estimated rate of missed appointments of only $5 \%$ [12]. If any abnormality was detected, a total body computed tomography (CT) scan was performed, to obtain an objective morphologic assessment. Of these 602 patients, 63 developed histologically or cytologically proven metastases to the liver as primary extra-ocular metastatic site, representing a rate of $10.5 \%$ during the 14 years of the study period. The 3- and 5-year cumulative risk of metastases were 5.8\% (SE 2.4\%) and $17.3 \%$ (SE 3.7\%), respectively. None of these 63 patients was lost to follow-up. All these patients were consecutively treated at Léon Bérard Cancer Centre in Lyon, France, until January 1, 2003.

All 63 patients with diagnosis of liver metastases from uveal melanoma underwent a multidisciplinary treatment program and were seen by the same surgeon to assess the question of surgical removal of liver metastases. Patients with diffuse metastases on the CT scan were not operated on and received systemic chemotherapy or best supportive care (meaning no specific therapy) only. Other patients underwent laparotomy. Selection criteria for liver resection were as follows: (1) medical fitness, (2) no signs of disseminated disease, and (3) tumor anatomically confined to the liver with $30-40 \%$ of normal parenchyma that could be preserved after complete tumor resection. When complete resection was not possible because of diffuse liver involvement (miliary metastatic liver disease seen at opensky surgery and/or intraoperative ultrasonography), partial debulking surgery was performed followed by implantation of an intra-arterial catheter for postoperative chemotherapy. Each time it was possible, patients underwent complete liver metastasis resection, implantation of an intra-arterial catheter and postoperative intra-arterial chemotherapy using fotemustine $\left(100 \mathrm{mg} / \mathrm{m}^{2}\right.$ infused over $4 \mathrm{~h}$ every week or every 2 weeks in case of platelet decrease, over $2-4$ months $)$ or cisplatin $\left(80 \mathrm{mg} / \mathrm{m}^{2}\right.$ infused over $4 \mathrm{~h}$ every week or every 2 weeks in case of platelet decrease, over 2-4 months).

\section{Methods}

Medical records were reviewed for all patients to determine the prognostic factors that significantly influenced survival after first diagnosis of liver metastases. The following information was collected: gender, age at uveal melanoma treatment and age at metastasis treatment, performance status (Karnofsky index), anatomic location, TNM tumor categories and pathologic data of the primary uveal melanoma when available, type of uveal melanoma treatment, ocular recurrence, sites of systemic metastases, time interval between initial tumor treatment and diagnosis of metastases (called metastasis-free interval), symptoms from metastases, metastasis diagnosis, LFTs (including serum aspartate and alanine aminotransferase, alkaline phosphatase and lactate dehydrogenase levels), percentage of hepatic replacement, number of liver metastases, largest liver metastasis diameter, morbidity and mortality related to metastasis treatment, and overall survival period after uveal melanoma treatment and after metastasis diagnosis. The performance status is classically categorized into three groups: essentially asymptomatic patients (Karnofsky index 10090; equivalent to Eastern Cooperative Oncology group (ECOG) 0 [17]], symptomatic patients [Karnofsky index 80-60; ECOG 1-2), and symptomatic patients with poor performance status (Karnofsky index 50-10; ECOG 3-4). The classification of uveal melanomas was obtained using tumor thickness and largest tumor diameter (LTD). Patients were allocated into three groups according to the "TNM Classification of Malignant Tumors" (systematized nomenclature of medicine ICD-O C69.3,4) [31]: patients with T1 tumors ( $\leq 2.5 \mathrm{~mm}$ in thickness and $\leq 10 \mathrm{~mm}$ in LTD), patients with T2 tumors $(>2.5$ and $\leq 10 \mathrm{~mm}$ in thickness and $>10$ and $\leq 16 \mathrm{~mm}$ in LTD) and patients with T3 tumors ( $>10$ 
$\mathrm{mm}$ in thickness and/or $>16 \mathrm{~mm}$ in LTD). This international classification is almost equivalent to that used by the COMS group, T1 corresponding to COMS "small" tumors, T2 to "medium-sized" ones and T3 to "large" ones $[32,33]$. Tumors including the ciliary body are termed hereafter "ciliary body location" in this report.

\section{Statistical assessment}

Overall patient survival time was calculated from the date of diagnosis of liver metastases to the time of death. Observations relating to patients still alive on January 1, 2003 were censored after this date. Survival and time-toevent rates, with standard error (SE), were calculated according to the Kaplan-Meier method and compared using the log rank test $[9,19]$. On this basis, curves were constructed that depicted the probability of survival per month following diagnosis of metastases. Moreover, we completed the univariate analysis by a univariate Cox regression model, indicating $P$-values, risk rates and 95\% confidence intervals in Table 3. A $P$-value below 0.05 was considered to be statistically significant. Multivariate analysis, to evaluate the independence of prognostic factors, utilized the Cox proportional hazards model, with significance of the parameters estimated by the likelihood ratio test [4]. Relative rates were calculated including 95\% confidence intervals. The variables tested in Cox regression fulfilled the assumption of proportional hazards.

\section{Results}

Patient characteristics and follow-up evaluation

Of the 63 patients with liver metastases identified during the study period, 30 were men and 33 women. The median age was 57 years (range 28-81) at initial eye treatment and 61 years (range 31-85) at liver metastasis diagnosis. Fiftythree patients died within the study period, with a median time of 13 months (range 2.5-110) from diagnosis of liver metastases to death. Thirty-eight patients $(60 \%)$ survived more than 1 year and $17(27 \%)$ more than 2 years. Ten patients were alive at the time of analysis; seven had metastatic recurrence whereas three had no evidence of disease. The median follow-up of these ten survivors was 29 months (range 16-110).

\section{Primary uveal melanoma}

The anatomic locations of primary uveal melanoma tumors were 44 choroidal, 18 ciliary or ciliochoroidal, one iridociliochoroidal tumors. Ocular tumor classification, including thickness and LTD, is presented in Table 1. Initial and further treatments for uveal melanoma are described in
Table 1 Ocular tumor characteristics of 63 consecutive patients treated for liver metastases from primary uveal melanoma

\begin{tabular}{lcc}
\hline Ocular tumor characteristics & Number & Proportion (\%) \\
\hline Ciliary body involvement $^{2}$ & 19 & 30.2 \\
Categories (according to TNM classification) $^{\mathrm{a}}$ & \\
T1 & 4 & 6.3 \\
T2 & 35 & 55.6 \\
T3 & 16 & 25.4 \\
Thickness (mm) & \\
$\leq 5$ & 18 & \\
$>5$ & 38 & 60.3 \\
$\leq 10$ & 45 & 71.4 \\
$>10$ & 11 & 17.5 \\
Largest basal tumor diameter $(\mathrm{mm})^{\mathrm{c}}$ & \\
$\leq 10$ & 19 & 30.2 \\
$>10$ & 36 & 57.1 \\
\hline
\end{tabular}

${ }^{\mathrm{a}}$ Missing value for 8 cases

${ }^{\mathrm{b}}$ Missing value for 7 cases

${ }^{\mathrm{c}}$ Missing value for 8 cases

Table 2. Fifteen patients (24\%) with subsequent regrowth of their uveal melanoma were retreated. Enucleation was necessary in 21 patients. It was the first treatment in eight patients; in other cases, it was consecutive to tumor regrowth (eight patients), painful blindness with neovascular glaucoma (five patients), complete retinal detachment associated with visual loss (one patient) or scleral necrosis (one patient). Two patients had two causes. Pathologic data, were available for 19 patients, showed the following distribution: three patients presented with epithelioid cells, five with spindle cells, seven with mixed-cells and four with total tumor necrosis.

\section{Liver metastases}

All but one patient were asymptomatic at the time of liver metastasis diagnosis. Karnofsky index was known for all patients, of whom $62(98.4 \%)$ scored $100-90$, and one

Table 2 Ocular treatments over time

\begin{tabular}{lrrrrr}
\hline & $\begin{array}{l}\text { Proton } \\
\text { beam } \\
\text { therapy }\end{array}$ & Brachytherapy & Enucleation & Other & Total \\
\hline 1st treatment & 38 & 12 & 8 & 5 & 63 \\
2nd treatment & 3 & 4 & 8 & 3 & 18 \\
3rd treatment & 1 & & 5 & 1 & 7 \\
Total & 42 & 16 & 21 & 9 & \\
\hline
\end{tabular}

The "Brachytherapy" group included treatment with ruthenium plaque or iridium

The "Other" group included transpupillary thermotherapy or surgical removal of the tumor except enucleation 
(1.6\%) scored 80. The metastatic work-up at the time of diagnosis of extra-ocular metastases showed the following: 54 patients had metastases confined to the liver and nine had metastases in the liver and at other sites, including lung in five patients, bones in three, skin, pancreas and lymph nodes in one. Seventeen patients $3 \%$ of the screened population, $27 \%$ of the metastatic population) presented ten or fewer liver metastases on the preoperative CT scan, with a median number of three. All but one patient had less than $25 \%$ of hepatic replacement by the tumor disease. This patient was the only one in the series to have a more than 3 $\mathrm{cm}$ in diameter metastasis and abnormal LFTs.

The time interval between uveal melanoma treatment and metastasis diagnosis ranged from 0 (4 patients) to 145 months (median time 29 months). Thirty-nine patients $(62 \%)$ developed liver metastases within 3 years. It took longer than 5 years for 12 patients (19\%), and even longer than 10 years for two patients.

\section{Metastasis treatment procedures}

Twenty-eight patients $(4.7 \%$ of the screened population and $44.4 \%$ of the metastatic population) underwent liver surgery. At direct examination and intraoperative ultrasonography, 14 patients were found to have diffuse miliary metastatic liver disease that was missed by preoperative imaging. They underwent partial metastatic debulking then received postoperative intra-arterial chemotherapy with fotemustine or cisplatin (mean 8 cycles, range 4-14 cycles). Fourteen patients $(2.3 \%$ of the screened population and $22.2 \%$ of the metastatic population) had complete removal of liver metastases and 11 of these received postoperative intra-arterial chemotherapy with fotemustine or cisplatin (mean 7 cycles, range $2-12$ cycles). No significant surgical complication was experienced. The remaining 35 patients with major liver involvement or diffuse metastases (nine patients) after initial work-up were not operated on. They received best supportive care (9 patients) or systemic chemotherapy (26 patients). Chemotherapy consisted of an association of cisplatin, vinblastine and dacarbazine (CVD), and in eight patients, dacarbazine alone in 14 patients and interferon \pm interleukin 2 in four patients.

\section{Survival after liver metastases diagnosis}

The median survival for the 63 patients was 47 months (range 3-190 months) after primary uveal tumor treatment, and 15 months (range 3-110 months) after liver metastasis diagnosis. The overall survival rate after metastasis diagnosis was 59\% (SE 6\%) at 1 year, $29 \%(\mathrm{SE} 6 \%$ ) at 2 years and $13 \%(\mathrm{SE} 5 \%)$ at 4 years (Fig. 1). Median overall survival after metastasis diagnosis reached 25 months (range 11-110 months) for the 14 patients who underwent complete resection, 16 months (range 3-35 months) for the

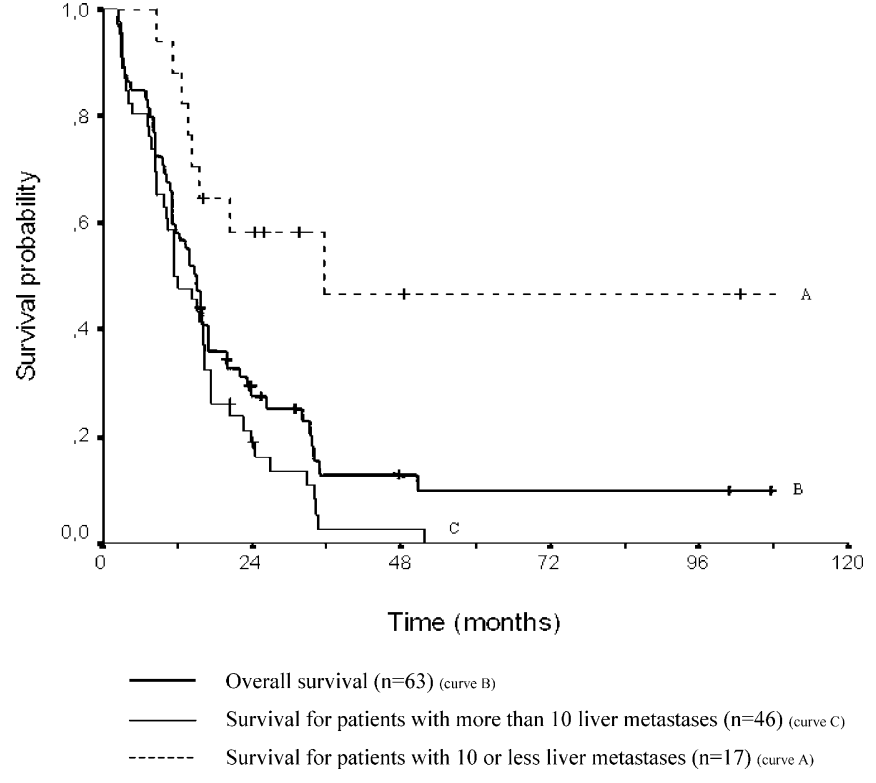

Fig. 1 Overall survival after diagnosis of liver metastases for all patients and according to the preoperative number of liver metastases $(P=0.0002)$-Kaplan-Meier estimator $(n=63)$

14 patients who underwent debulking surgery and 11 months (range 3-52 months) for the 35 patients who received systemic chemotherapy or best supportive care (Fig. 2).

Predictors of survival in univariate analysis

(Table 3, univariate Cox regression model)

\section{Patient demographics}

Survival was not influenced by age $(P=0.06)$ or by gender $(P=0.69)$ in the univariate analysis. Patients below the age

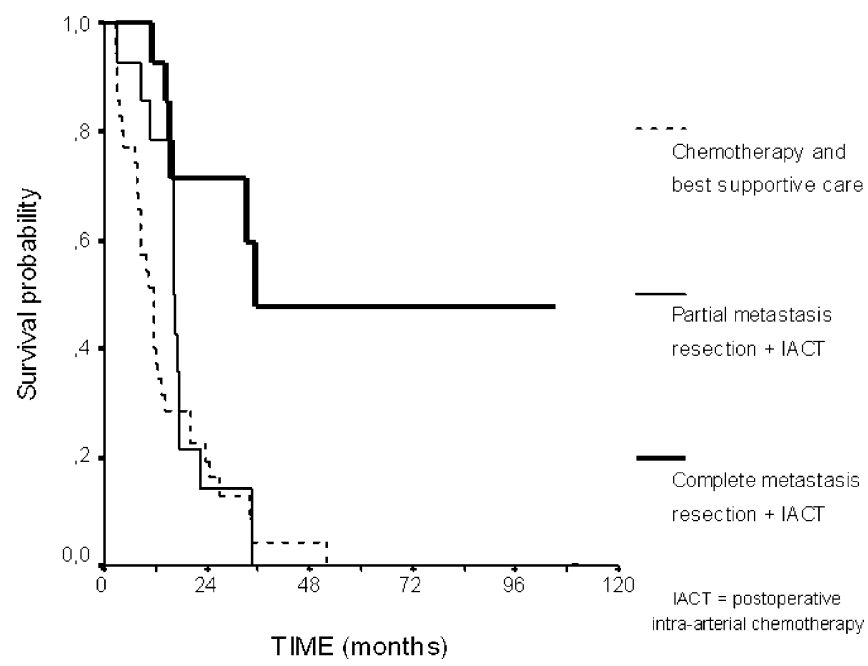

Fig. 2 Overall survival after diagnosis of liver metastases according to metastasis treatment $(P=0.0002)$-Kaplan-Meier estimator $(n=63)$ 
Table 3 Univariate predictors of outcome (univariate Cox regression model)

\begin{tabular}{|c|c|c|c|c|}
\hline$\underline{\text { Variable }}$ & Number of patients & Risk Rate & $95 \% \mathrm{CI}^{\mathrm{a}}$ & $P$ \\
\hline Overall & 63 & & & \\
\hline \multicolumn{5}{|l|}{ Sex } \\
\hline Male & 30 & 1.00 & & \\
\hline Female & 33 & 0.89 & $0.52-1.55$ & 0.69 \\
\hline \multicolumn{5}{|l|}{ Age at metastases diagnosis } \\
\hline$\leq 70$ & 48 & 1.00 & & \\
\hline$>70$ & 15 & 1.84 & $0.99-3.39$ & 0.06 \\
\hline \multicolumn{5}{|l|}{ Primary tumor location } \\
\hline Non ciliary body & 19 & 1.00 & & \\
\hline Ciliary body & 44 & 1.70 & $0.92-3.11$ & 0.09 \\
\hline \multicolumn{5}{|l|}{ Primary tumor TNM } \\
\hline $\mathrm{T} 1$ or $\mathrm{T} 2$ & 15 & 1.00 & & \\
\hline $\mathrm{T} 3$ & 38 & 1.10 & $0.57-2.14$ & 0.78 \\
\hline \multicolumn{5}{|l|}{ Primary tumor thickness } \\
\hline$\leq 5 \mathrm{~mm}$ & 18 & 1.00 & & \\
\hline$>5 \mathrm{~mm}$ & 38 & 0.94 & $0.50-1.78$ & 0.85 \\
\hline \multicolumn{5}{|l|}{ Primary tumor diameter } \\
\hline$\leq 10 \mathrm{~mm}$ & 19 & 1.00 & & \\
\hline$>10 \mathrm{~mm}$ & 36 & 1.04 & $0.55-1.95$ & 0.91 \\
\hline \multicolumn{5}{|l|}{ Primary tumor treatment (1) } \\
\hline No enucleation & 49 & 1.00 & & \\
\hline Enucleation & 8 & 1.07 & $0.93-1.22$ & 0.41 \\
\hline \multicolumn{5}{|l|}{ Primary tumor treatment (2) } \\
\hline Proton beam therapy & 32 & 1.00 & & \\
\hline Brachytherapy & 12 & & & \\
\hline Thermotherapy or surgery & 5 & 1.09 & $0.96-1.25$ & 0.21 \\
\hline Enucleation & 8 & & & \\
\hline \multicolumn{5}{|l|}{ Ocular recurrence } \\
\hline Recurrence & 15 & 1.00 & & \\
\hline No recurrence & 48 & 0.94 & $0.48-1.83$ & 0.85 \\
\hline \multicolumn{5}{|l|}{ Disease-free interval } \\
\hline$\leq 24$ months & 26 & 1.00 & & \\
\hline$>24$ months & 37 & 0.96 & $0.55-1.66$ & 0.87 \\
\hline$\leq 60$ months & 51 & 1.00 & & \\
\hline$>60$ months & 12 & 1.62 & $0.83-3.19$ & 0.18 \\
\hline \multicolumn{5}{|l|}{ Liver metastases treatment } \\
\hline Surgery & 14 & 1.00 & & \\
\hline Intra-arterial chemotherapy & 14 & 1.22 & $1.10-1.37$ & 0.00002 \\
\hline Systemic chemotherapy or BSC & 35 & & & \\
\hline \multicolumn{5}{|l|}{ Number of liver metastases } \\
\hline$\leq 10$ & 17 & 1.00 & & \\
\hline$>10$ & 46 & 4.02 & $1.85-8.73$ & 0.00006 \\
\hline
\end{tabular}

of 70 years had a 2-year overall survival of $34 \%$ (SE 7\%) compared with 7\% (SE 6\%) for patients older than 70 years. Performance status was not statistically significant, since all but one patient belonged to the same group (Karnofsky index 100-90) (Table 3).

\section{Characteristics of the uveal tumor}

The categories of primary uveal melanoma (T1/T2 versus T3, $P=0.78)$, including thickness $(\leq 5 \mathrm{~mm}$ versus $>5 \mathrm{~mm}$, $P=0.85 ; \leq 10 \mathrm{~mm}$ versus $>10 \mathrm{~mm}, P=0.89)$ and LTD $(\leq 10$ $\mathrm{mm}$ versus $>10 \mathrm{~mm}, P=0.91)$, as well as initial ocular treatment $(P=0.21)$ and ocular recurrence $(P=0.85)$ were 
not predictive of outcome in our series. Conversely, the anatomic location of the primary uveal melanoma seemed to influence outcome, in that melanomas involving the ciliary body had a 2-year overall survival of $11 \%$ (SE 7\%) compared to $39 \%$ (SE $8 \%$ ) for strictly choroidal melanomas $(P=0.09)$ (Fig. 3).

\section{Presentation and treatment of liver metastases}

Neither a metastasis-free interval of less than 24 or 60 months after primary tumor treatment was predictive of outcome ( $P=0.87$ and $P=0.18$, respectively). Overall survival was also not associated with LFTs in this series, since all but one patient presented normal levels. For the same reasons, the percentage of hepatic replacement or the largest metastasis diameter were not predictive of outcome in this series. In contrast, the number of liver metastases detected preoperatively (up to ten metastases) was a strong indicator of outcome $(P=0.00006)$ (Fig. 1). All 5-year survivors in this study had maximally ten liver metastases preoperatively uncovered. Ten $(59 \%)$ of the 17 patients with ten or fewer liver metastases belonged to the complete liver metastases resection group, versus only four $(9 \%)$ of the 46 patients with more than ten liver metastases.

Lastly, complete resection of liver metastases noticeably influenced outcome $(P=0.00002)$ (Fig. 2). There was no survival difference between the group treated by partial metastasis resection and the group treated by systemic chemotherapy or best supportive care $(P=0.32)$.

\section{Multivariate analysis of outcome (Table 4)}

In a multivariate regression model including the number of preoperative metastases, ciliary body location and age, the number of metastases and ciliary body location were independently associated with survival $(P<0.0001$ and $P=$ 0.017 , respectively). Age was treated as a continuous variable in the multivariate analysis only. In this model, the

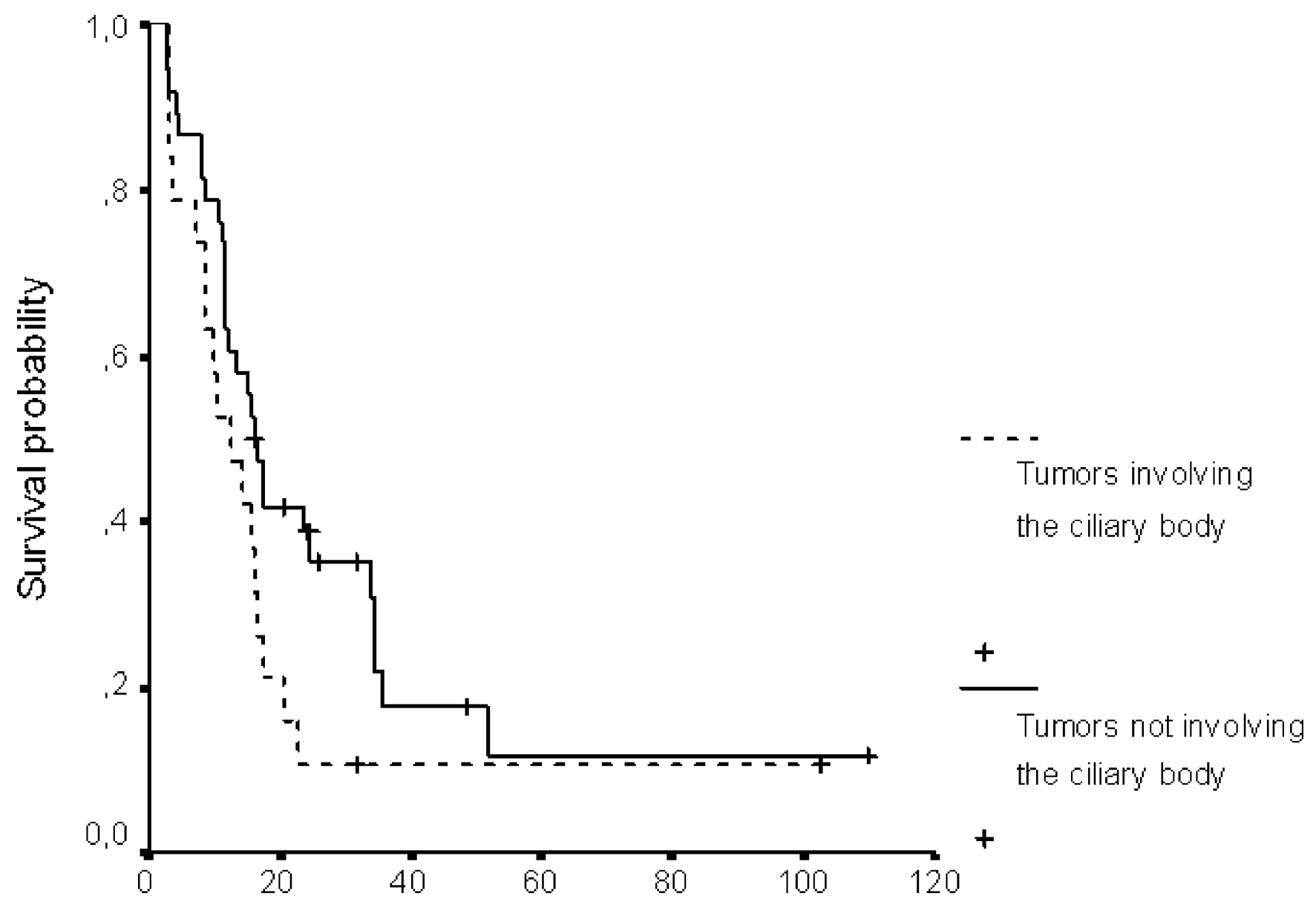

\section{Time (months)}

Fig. 3 Overall survival (after metastases treatment) according to uveal melanoma location $(P=0.084)$-Kaplan-Meier estimator $(n=63)$ 
Table 4 Overall survival of 63 incident cases of uveal melanoma with liver metastases

\begin{tabular}{|c|c|c|c|c|c|}
\hline Factor & Category & Number & Risk rate & $95 \% \mathrm{CI}^{\mathrm{a}}$ & $P^{\mathrm{b}}$ \\
\hline \multirow[t]{2}{*}{ Number of metastases } & $\leq 10$ & 17 & 1.00 & & \\
\hline & $>10$ & 46 & 4.98 & $2.1-11.6$ & $<0.0001$ \\
\hline \multirow[t]{2}{*}{ Melanoma location } & Not involving ciliary body & 44 & 1.00 & & \\
\hline & Involving ciliary body & 19 & 2.20 & $1.2-4.1$ & 0.017 \\
\hline Age (decades) & Linear $^{\mathrm{c}}$ & $\mathrm{NA}^{\mathrm{d}}$ & 1.01 & $0.99-1.04$ & 0.285 \\
\hline
\end{tabular}

Risk rates of death associated with number of metastases, ciliary body location and age (multivariate Cox model)

${ }^{\mathrm{a}} \mathrm{CI}=$ confidence interval

${ }^{\mathrm{b}}$ Likelihood ratio test for heterogeneity

${ }^{\mathrm{c}}$ Age was treated as a continuous variable. The relative rates in the table were taken from the linear model. Quadratic terms were also tested and were not significant

${ }^{\mathrm{d}} \mathrm{NA}=$ not applicable

treatment of metastases weighted similarly to the number of preoperative metastases because of the high correlation between the two variables. Indeed, the number of metastases directly influenced the choice of liver metastasis treatment (Table 4).

\section{Discussion}

The prognosis of metastatic uveal melanoma is poor. Metastases to the liver are the life-limiting component of the disease in the majority of patients $[10,11,23,27]$. Thus, median survival is 19 months for metastatic patients from uveal melanoma with no liver involvement, but falls to 7 months after liver dissemination [11]. A few prognostic factors have been identified to date, but they still remain poorly understood. Only four studies have provided a multivariate analysis of these factors $[3,5,6,13]$. To sum up, age above 50/55 years, male gender, short interval of time between uveal melanoma diagnosis and metastasis diagnosis, and severity of liver metastatic burden (poor performance status, elevated percentage of hepatic replacement, large metastasis diameter, and abnormalities in LFTs) have been shown to have a negative impact on survival [3, $5,6,13,23]$. The limitation of most of these studies is that they refer to limited numbers of metastatic patients, which does not fit with complex regression models such as the Cox proportional hazards model $[2,11,23]$. Additionally, these studies may suffer from selection biases, i.e. age [13, 25], metastasis-free interval or metastasis location [1]; therefore, results may not directly be compared or generalized. Beside our series, only two studies with no obvious bias regarding patient selection have been reported $[5,6]$. In these studies, however, metastasis treatment was mainly supportive care or systemic chemotherapy, which is well known to be insufficient in uveal melanoma metastases $[3,6,13]$. Surgery was carried out in only $1-9 \%$ of the cases, compared with $44 \%$ in our series. The two reports showed that patients diagnosed during screening examination survived significantly longer $[5,6]$. There is no evidence that screening examination improves the outcome; nevertheless, it seems nowadays advocated for all patients affected by uveal melanoma [5, 6]. Since 1983, all our patients have been followed semi-annually by clinical examination and liver ultrasonography. As a consequence, all but one patient diagnosed with liver metastasis was asymptomatic, had good performance status, low hepatic replacement, and normal LFTs. This justified conducting the present study to determine new prognostic factors, applicable to metastatic uveal melanoma patients selected by regular screening.

The strength of our study is that the series is populationbased and consecutive. All 602 patients affected by uveal melanoma evaluated during the 14-year study period originated from a single centre (Croix-Rousse Hospital) and all metastatic patients (63) were referred to a single institution (Léon Bérard Cancer Center) in order to minimize physician-associated biases. This resulted in a quite significant number of operated-on patients. We have tried to define preoperative selection criteria, thanks to systematic CT scan. The two main limitations of our study are the retrospective collection of data and the wide variety of therapy protocols used thus precluding any definitive conclusion on the treatment efficacy and indication. However, data were collected from medical charts and systematic CT scan reviewing, which leaves little scope for error. In addition, we limited the number of variables studied in the Cox model according to sample size. Despite these limitations, we believe that our results may be extrapolated to the treatment of other patients with liver metastatic uveal melanoma. The metastatic population was not different in term of primary tumor characteristics from our whole population $[12,24]$.

In our series, $10.5 \%$ of the screened patients (63 of 602 ) were found to have liver metastases. Twenty-eight of them (4.7\% of the screened population and $44.4 \%$ of the metastatic population) were selected for liver surgery and postoperative intra-arterial chemotherapy. Fourteen patients presented intraoperative miliary metastatic liver disease, that was too small to be picked up by conventional imaging. Finally, only 14 of these patients $(2.3 \%$ of the screened population and $22.2 \%$ of the metastatic population) were able to undergo complete resection of liver metastases 
without any major surgical complication. This selection was based on the preoperative number of liver metastasis, which seems to be a good indicator of severity of metastatic burden. These patients consequently survived for a median time of 25 months $(P=0.0002)$, whereas the median reported survival following detectable liver metastasis dissemination is only $2-9$ months $[5,27]$. Although these results are somewhat disappointing according to the small number of patients with benefit, this screening program is considered nowadays as the most efficient and cost effective. Furthermore, these results suggest that there is a subgroup of metastatic patients who can benefit from aggressive surgery. Lastly, more intensive work-up with systematic use of staging laparoscopy may be of value to improve liver assessment and patient selection, and to avoid unnecessary laparotomy.

We individualized new prognostic factors in a multivariate analysis. When building the regression model, we tried to take into account different aspects relevant to the clinical situation. To our knowledge, the influence of uveal melanoma characteristics (TNM tumor categories, tumor thickness, LTD, location of primary uveal tumor and uveal melanoma recurrence) on the survival of metastatic patients has not been previously reported. LTD, which is known to be the most important clinical risk factor for the development of metastases among patients treated for primary uveal melanoma [7, 15, 26, 28], is no longer of prognostic relevance once metastases have occurred. In contrast, ciliary body involvement, also known to worsen the prognosis in patients affected by uveal melanoma [16, 22, 26], irrespective of tumor size, remained an independent predictor of death after metastases occurrence in our series. Tumors involving the ciliary body are recognized to be more likely to harbor monosomy of chromosome 3 and trisomy of chromosome $8 \mathrm{q}$, a situation that is associated with a very high risk of death $[14,20]$. Clearly, tumor genetics may represent an as yet unexplored confounder of prognosis. Furthermore, we found that the preoperative number of liver metastases was a significant independent predictor of survival in our series. Patients with more than
10 metastases had a 4.8 times increased risk of death. This threshold was arbitrarily chosen, in a will of simplification, since this has no real signification on a disease biology stand-point. Most of the patients had a smaller number of metastases. The median number of preoperative metastases in the subgroup of patients with fewer than ten metastases was only three, and patients with more than ten were usually found to have a huge number. Among other factors, this discrimination level should be used to select candidate patients for surgery and multimodal treatment. Indeed, if ten or fewer liver metastases are identified preoperatively, laparotomy can therefore be attempted.

We postulate that our results would not have been achieved without a systematic semi-annual hepatic screening that we therefore strongly advocate, since 1983, for all patients suffering from uveal melanoma. More frequent follow-up might be considered in high-risk patients, i.e. those with ciliary body involvement. Nevertheless, semiannual screening ultrasound clearly was ineffective for early detection of hepatic metastasis in most patients with uveal melanoma. It is far more likely that tumor biology and patient selection govern final outcome, rather than screening ultrasound. Moreover, our present study was not designed to assess the effectiveness of any treatment method, since therapy was not randomized. Nevertheless, there is clearly a need for a rigorous selection of patients candidates for surgical treatment [25] since our data show that complete surgical removal of metastases associated with postoperative intra-arterial chemotherapy seems to be effective for prolonging survival in a limited selected group of patients $(2.3 \%$ of the screened population and $22.2 \%$ of the metastatic population). Since no efficient medical treatment is yet available for metastatic patients, these data could serve as a basis for a randomized clinical trial comparing non-surgical standard treatment and surgery in patients with ten or fewer liver metastases. Better screening tests and more effective multimodality treatments are required to improve survival in a larger number of uveal melanoma patients with liver metastases.

\section{References}

1. Aoyama T, Mastrangelo MJ, Berd D, Nathan FE, Shields CL, Shields JA, Rosato EL, Rosato FE, Sato T (2000) Protracted survival after resection of metastatic uveal melanoma. Cancer 89:1561-1568

2. Bedikian AY, Kantarjian H, Young SE, Bodey GP (1981) Prognosis in metastatic choroidal melanoma. South Med J 74:574-577
3. Bedikian AY, Legha SS, Mavligit G, Carrasco $\mathrm{CH}$, Khorana S, Plager C, Papadopoulos N, Benjamin RS (1995) Treatment of uveal melanoma metastatic to the liver: a review of the M. D. Anderson Cancer Center experience and prognostic factors. Cancer 76:1665-1670

4. Cox D (1972) Regression models and life-tables (with discussions). J R Statist Soc Series B 34:187-220
5. Eskelin S, Pyrhonen S, HahkaKemppinem M, Tuomaala S, Kivela T (2003) A prognostic model and staging for metastatic uveal melanoma. Cancer 97:465-475

6. Gragoudas ES, Egan KM, Seddon JM, Glynn RJ, Walsh SM, Finn SM, Munzenrider JE, Spar MD (1991) Survival of patients with metastases from uveal melanoma. Ophthalmology 98:383-389; discussion 90 
7. Gragoudas ES, Seddon JM, Egan KM, Glynn RJ, Goitein M, Munzenrider J, Verhey L, Urie M, Koehler A (1988)

Metastasis from uveal melanoma after proton beam irradiation.

Ophthalmology 95:992-999

8. Gragoudas ES, Seddon JM, Egan KM, Polivogianis L, Hsieh CC, Goitein M, Verhey L, Munzenrider J, AustinSeymour M, Urie M et al (1986) Prognostic factors for metastasis following proton beam irradiation of uveal melanomas. Ophthalmology 93:675-680

9. Kaplan E, Meier P (1958) Nonparametric estimation from incomplete observation. J Am Stat Assoc 53:457481

10. Karakousis CP, Temple DF, Moore R, Ambrus JL (1983) Prognostic parameters in recurrent malignant melanoma. Cancer 52:575-579

11. Kath R, Hayungs J, Bornfeld N, Sauerwein W, Hoffken K, Seeber S (1993) Prognosis and treatment of disseminated uveal melanoma. Cancer 72:2219-2223

12. Kodjikian L, Roy P, Rouberol F, Chauvel P, Jean-Louis B, Garweg J, Little R, Sasco A, Grange J (2004) Survival after proton-beam irradiation of uveal melanomas. Am J Ophthalmol 137:1002-1010

13. Leyvraz S, Spataro V, Bauer J, Pampallona S, Salmon R, Dorval T, Meuli R, Gillet M, Lejeune F, Zografos L (1997) Treatment of ocular melanoma metastatic to the liver by hepatic arterial chemotherapy. J Clin Oncol 15:2589-2595

14. Li W, Gragoudas ES, Egan KM (2000) Metastatic melanoma death rates by anatomic site after proton beam irradiation for uveal melanoma. Arch Ophthalmol 118:1066-1070
15. McLean IW, Foster WD, Zimmerman LE (1982) Uveal melanoma: location, size, cell type, and enucleation as risk factors in metastasis. Hum Pathol 13:123-132

16. McLean IW, Foster WD, Zimmerman LE (1977) Prognostic factors in small malignant melanomas of choroid and ciliary body. Arch Ophtalmol 95:48-58

17. Oken M, Creech R, Tormey D (1982) Toxicity and response criteria of the Eastern Cooperative Oncology Group. Am J Clin Oncol 5:649-655

18. Patel JK, Didolkar MS, Pickren JW, Moore RH (1978) Metastatic pattern of malignant melanoma. A study of 216 autopsy cases. Am J Surg 135:807-810

19. Peto R, Peto J (1972) Asymptotically efficient rank invariant test procedures. J R Statist Soc Series A 135:185-206

20. Prescher G, Bornfeld N, Hirche H, Horsthemke B, Jockel KH, Becher R (1996) Prognostic implications of monosomy 3 in uveal melanoma. Lancet 347:1222-1225

21. Pyrhonen S (1998) The treatment of metastatic uveal melanoma. Eur J Cancer 34(Suppl 3):S27-S30

22. Raivio I (1977) Uveal melanoma in Finland. An epidemiological, clinical, histological and prognostic study. Acta Ophthalmol Suppl 133:1-64

23. Rajpal S, Moore R, Karakousis CP (1983) Survival in metastatic ocular melanoma. Cancer 52:334-336

24. Rouberol F, Roy P, Kodjikian L, Gérard J, Jean-Louis B, Grange J (2004) Survival, anatomic and functional longterm results in choroidal and ciliary body melanoma after ruthenium brachytherapy (a 15 years experience with betarays). Am J Ophthalmol 137:893-900

25. Salmon RJ, Levy C, Plancher C, Dorval T, Desjardins L, Leyvraz S, Pouillart P, Schlienger P, Servois V, Asselain B (1998) Treatment of liver metastases from uveal melanoma by combined surgery-chemotherapy. Eur J Surg Oncol 24:127-130
26. Seddon JM, Albert DM, Lavin PT, Robinson N (1983) A prognostic factor study of disease-free interval and survival following enucleation for uveal melanoma. Arch Ophthalmol 101:1894-1899

27. Seregard S (1996) Posterior uveal melanoma. The Swedish perspective. Acta Ophthalmol Scand 74:315-329

28. Shammas HF, Blodi FC (1977) Prognostic factors in choroidal and ciliary body melanomas. Arch Ophthalmol 95:63-69

29. Shields J, Shields C (1992) Introduction to melanocytic tumors of the uvea. In: Shields J, Shields C (eds) Intraocular tumors; a text and atlas. Williams \& Wilkins, Philadelphia

30. Singh AD, Wang MX, Donoso LA, Shields CL, Potter PD, Shields JA, Elston RC, Fijal B (1996) Familial uveal melanoma, III. Is the occurrence of familial uveal melanoma coincidental? Arch Ophthalmol 114:1101-1104

31. Sobin L, Wittekind C (2002) TNM classification of malignant tumours. 6th ed. Wiley-Liss, Genève

32. The Collaborative Ocular Melanoma Study (2001) Assessment of metastatic disease status at death in 435 patients with large choroidal melanoma in the Collaborative Ocular Melanoma Study (COMS): COMS report no. 15. Arch Ophthalmol 119:670-676

33. The Collaborative Ocular Melanoma Study Group (1997) Factors predictive of growth and treatment of small choroidal melanoma: COMS report no. 5. Arch Ophthalmol 115:1537-1544

34. Vidal JL, Bacin F, Albuisson E, Rozan R, Desjardins L, D'Hermies F, Grange JD, Chauvel P, Caujolle JP, Sahel J et al (1995) "Melanoma 92". Epidemiological study of uveal melanoma in France. J Fr Ophtalmol 18:520-528 